Ann. Biol. anim. Bioch. Biophys., 1977, 17 (2), 137-146.

\title{
Effets de l'ingestion de tri-dodécanoate de glycérol (Tri C12 : 0) sur la composition en acides gras des lipides de différents dépôts adipeux chez le porc
}

\author{
par Y. DEMARNE, C. E. PERAZA-CASTRO**, Y. HENRY*, J. FLANZY \\ Station de Recherches de Nutrition, \\ * Station de Recherches sur l'Elevage des Porcs, \\ I.N.R. A., 78350 Jouy-en-Josas \\ ** Université Nationale Autonome de Mexico, \\ Unité de Xochimilco, \\ Division des Sciences Biologiques et Médicales, Mexico.
}

Summary. Effects on lipid fatty acid composition in different adipose deposits of pig ingesting glycerol tri-dodecanoate (Tri C12:0).

In this report we study the effects on the falty acid composition of lipids of the 30 to $90 \mathrm{~kg}$ growing pig ingesting a lipid-free diet and a diet containing 15 p. 100 of glycerol trilaurate (Tri C12:0). These effects are compared in 7 different adipose tissue samples at $90 \mathrm{~kg}$ liveweight.

Our results confirm that internal adipose tissue lipids are richer in saturated fatty acids than exterior deposit lipids. This observation is independent from the diet tested.

In our experimental conditions, lauric acid ( $1212: 0)$ always counts for less than 10 p. 100 of the total fatty acids found in adipose tissues. There is none of this fatty acid in the adipose tissues of animals fed the lipid-free diet. Lauric acid appears in stored lipids in association with myristic acid (C14:0) in amounts varying between 10 and 15 p. 100 of total fatty acids.

Exogenous supply of lauric acid does not change the quantities of palmitic acid (C16:0) in the different adipose tissue lipids. On the other hand, there is clearly less stearic acid (C18:0) than when a lipid-free diet is given.

The effects observed on unsaturated fatty acids vary depending on the fatty acid studied. For example, when glycerol irilaurate is ingested, almost iwice as less oleic acid (C18:109) is found in stored lipids. However, there is twice more palmitoleic acid (C16:1 107$)$ than when a lipid-free regimen is given.

In general, ingestion of glycerol irilaurate results in more saturated body lipids, that fatty acid having a depressive effect on oleic acid storage and causing a large amount of $\mathrm{C} 12$ and C14-saturated fatty acids to appear.

\section{Introduction.}

Chez le Porc, comme chez le Rat, l'ingestion prolongée d'un régime dépourvu de lipides conduit à la mise en réserve de triglycérides contenant environ 30 p. 100 
d'acide palmitique (C16:0) et 50 p. 100 d'acide oléique (C18:1 109) (Bollinger, 1963 ; Flanzy, François et Rérat, 1970 ; Raulin et Launay, 1964 ; Clément ef al., 1963).

Par rapport à ce qui est observé dans ces conditions nutritionnelles, l'introduction d'un mélange d'acides gras saturés à chaîne longue (C16:0 et C18:0) dans l'alimentation du jeune Rat en croissance ne modifie pas le rapport acides gras saturés/acides gras insaturés des triglycérides de réserve (Demarne et al., 1974). Même un apport massif de tripalmitate de glycérol (Tri C16:0) ne fait varier que dans de faibles proportions les concentrations en acides palmitique et oléique dans les lipides du tissu adipeux (Bollinger et Reiser, 1965). A la différence de ce qui est observé avec les acides gras insaturés (Clément et al., 1963 ; Demarne et al., 1975), les feneurs en acides gras longs et saturés dans l'adipocyte apparaissent donc comme étant peu influencées par les apports exogènes d'acides gras de ce type.

Chez le Rat, les effets nutritionnels des acides gras saturés à chaîne moyenne, normalement absents du tissu adipeux, varient avec la longueur de la chaîne. Ainsi, en cas d'ingestion prolongée d'acide caprylique $(C 8: 0)$, la composition en acides gras des triglycérides de réserve est identique à celle qui est observée en cas d'ingestion d'un régime lipidoprive (Bollinger ef Reiser, 1965 ; Kaunitz ef Johnson, 1968). En revanche, les acides gras ayant 10, 12 ou 14 atomes de carbone peuvent modifier la composition en acides gras des lipides corporels (Bézard et Bugaut, 1969 ; Bézard et Clément, 1964 ; Bollinger, 1963 ; Eckstein, 1929 ; Longenecker, 1939 ; Powell, 1930 ; 1932 ; Vischer, 1946). Par rapport aux acides gras à chaînes plus courtes, ils sont susceptibles de subir des élongations au niveau des microsomes el des mitochondries (Harlan et Wakil, 1963 ; Nutgeren, 1965 ; Wakil, 1961) et d'être oxydés moins rapidement (Bach, Mélais et Warter, 1968 ; Métais, Bach et Warter, 1967).

Chez le Porc, l'acide laurique (C12:0), qui représente 50 p. 100 des acides gras totaux de l'huile de coprah, se retrouve en faible quantité dans les tissus adipeux lorsque l'animal ingère cette matière grasse. Parallèlement, on observe une augmentation des teneurs totales en acides gras saturés et une diminution sensible des feneurs en acide oléique (Creswell et Brook, 1971 ; Christensen, 1963 ; Flanzy et al., 1970). Cependant, dans cette espèce animale, les propriétés nutritionnelles des acides gras à chaîne moyenne ont été moins étudiées que chez le Rat.

En tenant compte de ces différentes données, il nous a paru nécessaire de rechercher dans quelle mesure l'acide laurique pouvait être introduit sous forme de triglycéride homogène (trilaurate de glycérol) dans l'alimentation du Porc ef quelle était l'influence de l'ingestion de cet acide gras sur la composition en acides gras du tissu adipeux. Dans cet article, nous attirons tout particulièrement l'attention sur le contrôle exercé par l'acide laurique sur la mise en dépôt des autres acides gras dans différents tissus adipeux.

\section{Matériel ef méthodes.}

\section{Animaux ef régimes.}

L'expérience est réalisée avec 12 porcs de race Large White mâles castrés d'un poids vif moyen initial de $29 \mathrm{~kg}$. Les animaux, préalablement répartis par couples homogènes en fonction du poids et de l'âge, sont affectés à deux lołs I ef II 
comportant chacun 6 répétitions, suivant la méthode des blocs complets (6 animaux par lot). Ils sont élevés sur litière de paille dans des cages collectives munies de dispositifs d'alimentation individuelle. L'expérience se déroule pendant la croissance entre 30 et $90 \mathrm{~kg}$ de poids vif.

Les compositions des régimes sont rapportées sur le tableau 1. Le régime qui est fourni aux animaux du lot I est équilibré à 16 p. 100 de matières azotées totales

TABLEAU 1

Composition des régimes (valeurs exprimées en p. 100 par rapport à la matière sèche)

\begin{tabular}{|c|c|c|}
\hline Lots & I. - Témoin & II. - Trilaurine \\
\hline 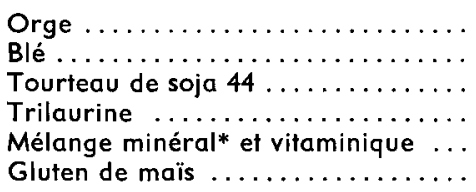 & $\begin{array}{l}39,5 \\
39,5 \\
18,0 \\
- \\
-\end{array}$ & $\begin{array}{r}24,5 \\
24,5 \\
25,5 \\
15,4 \\
3,8 \\
6,3\end{array}$ \\
\hline $\begin{array}{l}\text { Matières azotées totales } \\
\quad(\mathrm{N} \times 6,25 \mathrm{p} .100) \ldots \ldots \ldots \ldots \ldots \ldots \\
\text { Lysine }(\mathrm{p} .100) \ldots \ldots \ldots \ldots \ldots \ldots \\
\text { Energie digestible }(\mathrm{Kcal} / \mathrm{kg}) \ldots \ldots \ldots\end{array}$ & $\begin{array}{c}16,2 \\
0,78 \\
3160\end{array}$ & $\begin{array}{c}10,5 \\
0,97 \\
3725\end{array}$ \\
\hline
\end{tabular}

* Henry et Bourdon (1973).

ef ne contient qu'une faible quantité de lipides (environ 1 p. 100 de la matière sèche). Celui alloué aux animaux du lot II contient 15 p. 100 de trilaurate de glycérol. Sa composition est étudiée de façon à équilibrer le rapport matières azotées totales/ énergie digestible, ainsi que le rapport lysine/matières azotées totales (introduction de gluten de maïs en complément du tourteau de soja). D'après les valeurs de digestibilité rapportées pour l'acide laurique chez le Porc (Flanzy, Rérat ef François, 1968) ef la détermination de l'énergie brute des aliments, par rapport au régime de référence (lot I), l'introduction de trilaurate de glycérol dans la ration augmente l'apport d'énergie digestible d'environ 20 p. 100. C'est pourquoi on fixe, pour les animaux du lot II, un niveau de rationnement de 80 p. 100 par rapport à ceux du lot $I$.

Les compositions en acides gras des lipides totaux extraits des deux régimes sont rapportées sur le tableau 2. Les animaux reçoivent leurs régimes respectifs sous forme hydratée ( 2 parties d'eau pour une partie d'aliment), en deux repas quotidiens. On établit une échelle de rationnement évoluant en fonction du poids et qui passe pour les anımaux du lot I de $1,4 \mathrm{~kg} /$ jour à $30 \mathrm{~kg}$ à $3 \mathrm{~kg} / \mathrm{jour}$ au-delà de $80 \mathrm{~kg}$.

Prélèvement des tissus adipeux.

Les animaux des 2 lots sont abattus au poids de $90 \mathrm{~kg}$. On prélève des échantillons de tissu adipeux au moment de l'abattage à 5 niveaux différents. On examine : 
- le lard dorsal au niveau du dos (couches interne et externe) ;

- le lard dorsal au niveau du rein (couches interne et externe) ;

- le gras périrénal ou panne ;

- la poitrine ;

- les dépôts adipeux de la tête.

TABLEAU 2

Composition en acides gras des lipides ingérés (valeurs exprimées en p. 100 de l'ensemble des esters méthyliques dosés)

\begin{tabular}{|c|c|c|c|c|c|c|c|c|c|c|}
\hline Régimes & Lots & $\begin{array}{c}\text { Lipides } \\
\text { p. } 100\end{array}$ & $C 12: 0$ & C14:0 & $C 16: 0$ & C16:1 & C18:0 & C18:1 & $C 18: 2 \omega 6$ & C18: $3 \omega^{3}$ \\
\hline Témo & 1 & 1,2 & $<1,0$ & $<1,0$ & 21,7 & $<1,0$ & 1,8 & 15,9 & 54,6 & 5,3 \\
\hline Tri C12:0 & II & 15,4 & 92,4 & $<1,0$ & 1,4 & $<1,0$ & $<1,0$ & 1,8 & 3,5 & $<1,0$ \\
\hline
\end{tabular}

Méthodes analytiques.

Les lipides des tissus adipeux sont extraits à froid par le mélange chloroformeméthanol $(2: 1 \mathrm{v} / \mathrm{v})$ en suivant la technique proposée par Folch, Lees et SloaneStanley (1957).

La composition en acides gras des différents extraits lipidiques est obtenue par chromatographie en phase gazeuse. Les acides gras purifiés après saponification des différents extraits sont méthylés par le mélange méthanol-acide chlorhydrique (97: $3 \mathrm{v} / \mathrm{v})$. Ils sont ensuite séparés, puis dosés à l'aide d'un appareil CPG de marque Microtek Instr. Inc., muni d'un détecteur à ionisation de flamme. Les conditions opératoires sont les suivantes :

- colonne en acier inoxydable de $3 \mathrm{~m}$ de long et dont le diamètre intérieur est d'environ $3 \mathrm{~mm}$;

- support : chromosorb G.D.M.C.S. imprégné de 5 p. 100 de polymère E.G.S.S.X. ;

- température du four : $175^{\circ} \mathrm{C}$;

- nombre de plateaux théoriques : environ 2500 sur le pic de l'acide stéarique.

La surface des différents pics d'acides gras est obtenue grâce à l'emploi d'un intégrateur électro-mécanique Disc couplé avec l'enregistrement des chromatogrammes.

\section{Résultats et discussion.}

Croissance ef indice de consommation.

Les animaux du lot II acceptent bien le régime contenant 15 p. 100 de trilaurate de glycérol et l'échelle de rationnement initialement prévue est respectée. 
Les résultats des mesures de croissance et de consommation sont rapportés dans le tableau 3. Dans nos conditions expérimentales, les gains de poids moyens quotidiens ne sont pas significativement différents entre les 2 lots. L'indice de consommation, plus faible dans le lot II que dans le lot I, reflète les différences de concentration en énergie entre les deux régimes testés. En revanche, la quantité d'énergie digestible nécessaire pour obtenir $1 \mathrm{~kg}$ de gain de poids vif paraît supérieure d'environ 5,5 $\mathrm{p}$. 100 en cas d'ingestion de trilaurate de glycérol.

\section{TABLEAU 3}

Résultats généraux de croissance et de consommation alimentaire (valeurs moyennes indicatives)

Lots et régimes Lot I. - Témoin Lot II. - Tri C12:0

$\begin{array}{lc}\text { Poids moyen initial }(\mathrm{kg}) \ldots \ldots \ldots \ldots & 29,6 \\ \text { Poids moyen final }(\mathrm{kg}) \ldots \ldots \ldots \ldots \ldots & 91,8 \\ \text { Gain de poids }(\mathrm{g} / \text { jour }) \ldots \ldots \ldots \ldots \ldots & 647 \\ \text { Consommation moyenne }(\mathrm{g} / \text { jour) } \ldots & 290 \\ \text { Indice de consommation } \ldots \ldots \ldots \ldots & 3,57 \\ & \\ & \\ \text { férence statistiquement significative au seuil } P \leqslant 0,01 .\end{array}$

** Différence statistiquement non significative.

Composition en acides gras des dépôts adipeux.

Les résultats sont présentés dans le tableau 4. Afin de faciliter la discussion de ces valeurs numériques, nous les avons regroupées dans le tableau 5 par catégorie d'acides gras, d'une part en fonction de la longueur de chaîne et d'autre part en fonction du degré d'insaturation.

a) Effet de la localisation anatomique des dépôts adipeux.

Dans chaque lot, tous les tissus adipeux sous-cutanés étudiés présentent des concentrations en acides gras saturés moins élevées que les tissus adipeux profonds comme la panne. Ces différences résultent d'une augmentation des concentrations en acide palmitique $(\mathrm{C} 16: 0)$ et stéarique $(\mathrm{C} 18: 0)$ dans ce tissu.

Au niveau du lard dorsal, il existe également des différences de composition entre les couches externe et interne. Ainsi, on note que les concentrations en acides gras saturés sont plus élevées dans la couche adipeuse interne que dans la couche adipeuse externe. Cependant, ces différences paraissent plus accentuées chez les animaux ayant reçu le régime lipidoprive que chez ceux ayant ingéré le régime «Tri C12 ».

On n'observe pas de différences entre les tissus du lard dorsal selon qu'ils sont prélevés au niveau du dos ou au niveau du rain.

Parmi tous les tissus sous-cutanés prélevés, seul le tissu adipeux sous-cutané de la tête se différencie des autres par une concentration plus élevée en acides gras 
insaturés. Ces différences de composition en acides gras et plus particulièrement les variations du degré de saturation des lipides en fonction de leur localisation anatomique confirment les résultats de nombreux travaux antérieurs (Christensen, 1963 ; Christie et Moore, 1970 ; Hilditch et Williams, 1964 ; Ingr, 1971). L'ingestion quotidienne d'une quantité importante d'acide laurique pendant une longue période ne modifie pas cette donnée fondamentale.

\section{TABLEAU 4}

Compositions en acides gras des lipides extraits des différents tissus adipeux (valeurs moyennes exprimées en p. 100 des esters méthyliques dosés)

\begin{tabular}{|c|c|c|c|c|c|c|c|c|}
\hline Tissus adipeux & Lots & C12:0 & C14:0 & $\mathrm{C} 16: 0$ & C16:1 & C18:0 & $\mathrm{C} 18: 1$ & $C 18: 2 \omega 6$ \\
\hline \multirow{2}{*}{ Lard dorsal externe (dos) } & Témoin & $<1,0$ & 1,9 & 26,4 & 4,0 & 13,6 & 45,2 & 8,9 \\
\hline & $\overline{\text { TriC12:0 }}$ & 8,2 & 12,8 & 29,7 & 8,1 & 8,0 & 27,9 & 5,3 \\
\hline \multirow{2}{*}{ Lard dorsal interne (dos) } & Témoin & $<1,0$ & 1,9 & 28,8 & 2,8 & 17,3 & 43,1 & 6,1 \\
\hline & $\overline{\text { Tri C12:0 }}$ & 7,4 & 12,5 & 30,2 & 6,5 & 11,6 & 25,6 & 6,2 \\
\hline \multirow{2}{*}{ Lard dorsal externe (rein). } & Témoin & $<1,0$ & 2,3 & 26,9 & 3,1 & 16,0 & 44,4 & 7,3 \\
\hline & $\overline{\text { TriC12:0 }}$ & 7,4 & 13,5 & 27,8 & 8,9 & 9,0 & 28,6 & 4,8 \\
\hline \multirow{2}{*}{ Lard dorsal interne (rein) } & Témoin & $<1,0$ & $\overline{1,7}$ & 28,4 & 2,9 & 17,6 & 43,4 & 6,0 \\
\hline & $\overline{T r i C 12: 0}$ & 8,4 & 14,8 & 27,6 & 8,2 & 7,9 & 28,8 & 4,3 \\
\hline \multirow{2}{*}{ Sous-cutané (poitrine) } & Témoin & $\overline{<1,0}$ & 2,2 & 28,0 & 4,0 & 14,1 & 46,2 & 5,5 \\
\hline & $\overline{\text { TriC12:0 }}$ & 7,5 & 13,7 & 29,2 & 7,8 & 9,6 & 26,8 & 5,4 \\
\hline \multirow{2}{*}{ Sous-cutané (têłe) } & Témoin & $<1,0$ & 1,9 & 26,7 & 6,9 & 10,9 & 46,8 & 6,8 \\
\hline & Tri C12:0 & 6,3 & 11,2 & 27,7 & 7,2 & 9,9 & 31,4 & 6,3 \\
\hline \multirow{2}{*}{ Périrénal (Panne) } & Témoin & $<\overline{1,0}$ & 2,1 & 30,5 & 1,8 & 22,2 & 38,5 & 4,9 \\
\hline & Tri C12:0 & 8,3 & 14,4 & 33,1 & 4,6 & 13,9 & 20,9 & 4,8 \\
\hline
\end{tabular}

b) Effet du régime alimentaire.

Le remplacement d'une partie de l'apport énergétique du régime témoin par du trilaurate de glycérol entraîne de profonds changements au niveau de la composition en acides gras des 7 tissus adipeux étudiés.

Pour les différents acides gras saturés séparés et dosés, on note que :

- l'acide laurique (C12:0) est présent dans tous les tissus adipeux des animaux du lot II. Les concentrations selon les localisations anatomiques varient entre 6,0 ef 8,5 p. 100 de l'ensemble des acides gras, alors que cet acide n'est présent qu'à l'état de traces (1,0 p. 100) dans les lipides extraits des tissus correspondants chez les animaux ayant ingéré le régime lipido prive. L'acide laurique ne se retrouve pas dans 


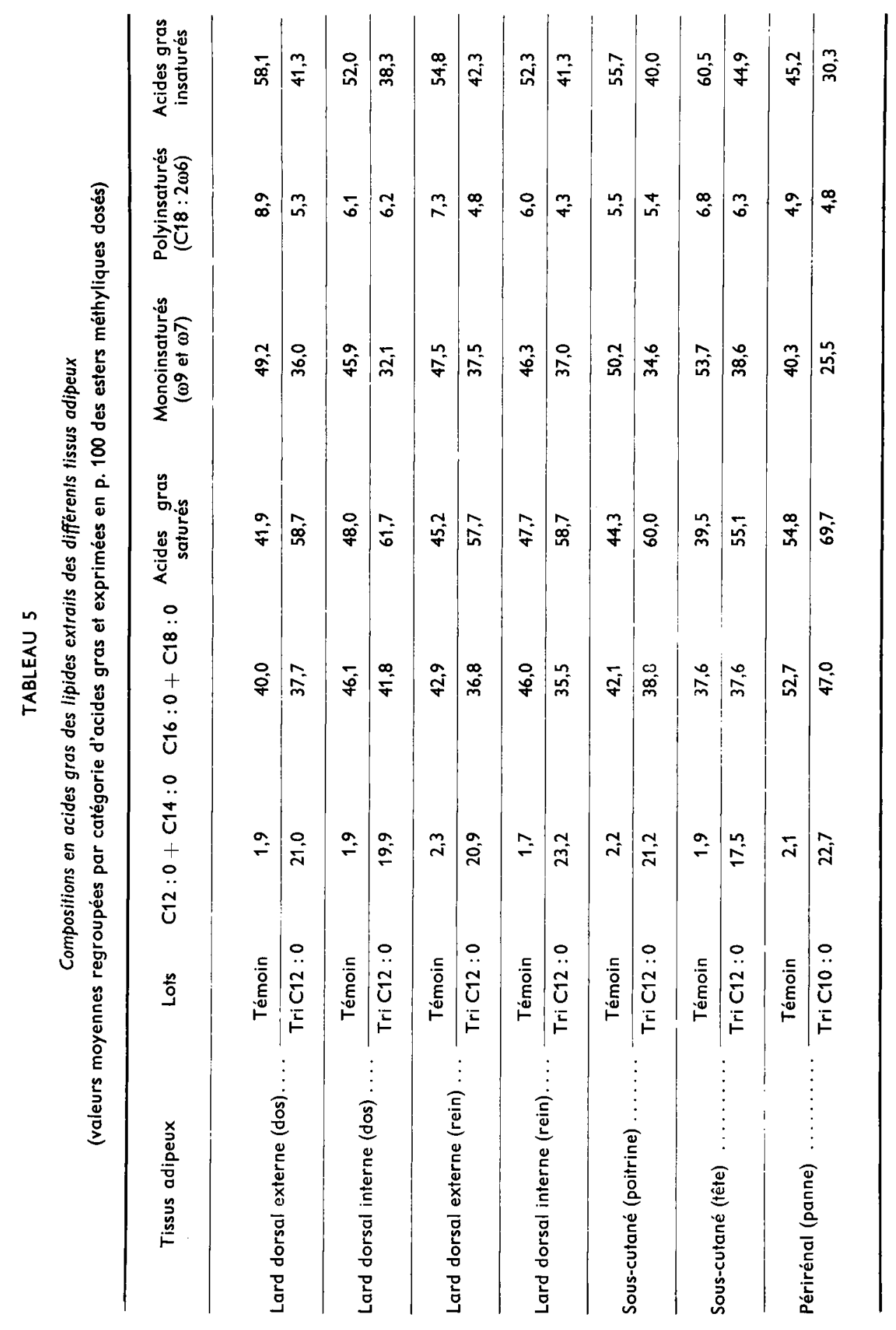


les tissus adipeux internes à des concentrations plus élevées que dans les différents tissus adipeux sous-cutanés examinés ;

- l'acide myristique (C14:0) qui représente entre 1,5 et 2,5 p. 100 de l'ensemble des acides gras dans le lot témoin est présent à des concentrations 6 à 7 fois supérieures dans le lot «Tri C12:0 »;

- les concentrations en acide palmitique $(\mathrm{C} 16: 0)$ ne sont pas significativement modifiées par la composition des régimes. Cependant, sur les 7 tissus adipeux analysés, 6 présentent des concentrations légèrement plus élevées en cas d'ingestion de trilaurate de glycérol ;

— l'acide stéarique (C18:0) qui représente entre 13 ef 22 p. 100 des acides gras totaux des différents tissus adipeux dans le lot témoin, ne représente plus que 8 à 14 p. 100 des acides gras totaux dans le lot «Tri C12:0 0 .

L'ingestion de trilaurate de glycérol par le Porc en croissance entraîne également de profondes modifications au niveau des proportions d'acides gras insaturés dans les différents dépôts adipeux. Ainsi, la proportion d'acide palmitoléique (C16:1) est presque toujours au moins doublée et la proportion d'acide oléique est fortement réduite : 20 à 32 p. 100 contre 38 à 47 p. 100 des acides gras tolaux. En revanche, la

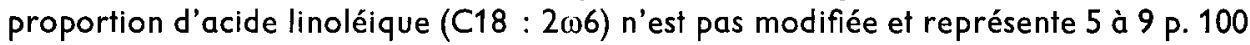
des acides gras totaux.

En se reportant aux résultats rapportés sur le tableau 5 , on remarque que l'ingestion prolongée de trilaurate de glycérol augmente très fortement les teneurs en acides gras saturés dans tous les tissus adipeux étudiés (55 à 70 p. 100 contre 40 à 55 p. 100 des acides gras totaux chez les animaux recevant le régime de référence). Ces résultats sont en accord avec ceux obtenus par Flanzy et al. (1970) avec de l'huile de coprah et démontrent d'une façon spécifique le rôle fondamental de l'acide laurique exogène dans l'orientation des biosynthèses d'acides gras au cours de la lipogénèse chez le Porc.

Cette augmentation des proportions d'acides gras saturés dans les dépôts ne provient pas d'une augmentation de la mise en réserve d'acides gras saturés à chaîne longue (acide palmitique et stéarique). Au contraire, dans 6 dépôts adipeux sur les 7 étudiés, les sommes des concentrations en acides gras de ce type (C16:0+C18:0) sont toujours plus faibles après ingestion de trilaurate de glycérol ; la différence provient dans ce cas d'une teneur plus faible en acide stéarique. L'accroissement des concentrations en acides gras saturés observé par rapport aux animaux de référence, ne résulte en fait que d'une augmentation des teneurs en acides gras saturés en $\mathrm{C} 12$ et en C14 variant selon les tissus considérés entre 16 et 22 p. 100 (données absolues).

Par rapport aux quantités importantes qui ont été ingérées au cours de la croissance, l'acide laurique n'est pas directement introduit en forte proportion dans le tissu adipeux. Par comparaison avec un autre acide gras également d'origine exogène comme l'acide linoléique (C18 : 2 $\omega 6$ ), son coefficient de stockage apparent est faible (Flanzy et ol., 1970).

L'introduction de trilaurate de glycérol pur dans la ration, à la différence d'huile de coprah (50 p. 100 de C12 : 0 et 15 à 20 p. 100 de C14:0), permet de définir les relations existant entre l'ingestion d'acide laurique ef l'apparition d'acide myristique dans les lipides de réserve. Les résultats présentés dans ce travail démontrent qu'il 
existe bien une élongation importante du type $\mathrm{C} 12: 0 \rightarrow \mathrm{C} 14: 0$ chez le Porc, puisque en cas d'ingestion de Tri C12 : 0 les teneurs en acide myristique sont toujours 6 à 7 fois plus importantes qu'en cas d'ingestion du régime de référence. Ces résultats sont à rapprocher de ceux présentés par Nutgeren en 1965 et qui démontraient que les enzymes des microsomes de Rat étaient susceptibles d'assurer in vitro l'élongation des acides gras à chaîne moyenne quand ils avaient au moins 10 atomes de carbone.

Dans l'alimentation du Porc, l'introduction d'acide laurique à des concentrations élevées apparaît donc comme un moyen efficace pour diminuer dans des proportions importantes les teneurs en acide oléique au niveau des réserves adipeuses. Cette méthode permet également d'augmenter le degré de saturation des lipides corporels, sans faire apparaître des quantités plus importantes d'acide palmitique et stéarique que celles obtenues par l'alimentation avec des régimes pauvres en lipides. Les acides laurique et myristique qui apparaissent dans les lipides de réserve ont des points de fusion élevés : respectivement $44^{\circ} \mathrm{C}$ et $54^{\circ} \mathrm{C}$ (Parisol, 1949). En conséquence, les points de fusion des lipides ainsi produits sont certainement beaucoup plus élevés que ceux qui sont classiquement observés avec une alimentation traditionnelle à base de céréales et de tourteau. Les caractéristiques technologiques et organoleptiques de ces matières grasses animales de composition originale mériteraient d'être étudiées.

Accepté en septembre 1976.

\section{Références}

BACH A., MÉTAIS P., WARTER J., 1968. Comparaison par l'étude du 14- $\mathrm{CO}_{2}$ de l'air expiré de l'utilisation des graisses à acides gras longs, moyens et courts. Influence du support. $C$. $R$. Soc. Biol., 162, 247-251.

BÉZARD J., BUGAUT M., 1969. Composition en triglycérides et en acides gras du tissu adipeux périrénal de rats recevant de l'huile de Coprah. Mobilisation sous l'influence du jeône et du froid. J. Physiol., 61, Suppl. 2, 224.

BÉZARD J., CLÉMENT J., 1964. Remaniements effectués au niveau digestif et au niveau des réserves adipeuses de la structure des triglycérides après administration d'un régime à base d'huile de coprah. C. R. Acad. Sc. Paris, 259, 3118-3120.

BOLLINGER J. M., 1963. The metabolism of fatty acids derived from dietary triglycerides. $P h$. D. Thesis, Texas A. and M. University, $74 \mathrm{p}$.

BOLLINGER J. M., REISER R., 1965. The metabolic fate of fatty-acids derived from diefary triglycerides. J. Am. Oil Chem. Soc., 42, 1130-1133.

CHRISTENSEN K. D., 1963. Various fatty acids in fat tissues of pigs of the Danish-Landrace breed fed with coconut fat or soy bean oil. Acta agric. Scand., 13, 249-254.

CHRISTIE W. W., MOORE J. H., 1970. The variation of triglycerides structure with fatty-acid composition in pig adipose tissue. Lipids, 5, 921-928.

CLÉMENT J., BOUCROT P., LORIETTE C., RAULIN J., 1963. Influence de la nature des lipides alimentaires sur la composition et la structure des triglycérides de réserve du rat blanc. Bull. Soc. Chim. Biol., 45, 1031-1042.

CRESWELL D. C., BROOKS C. C., 1971. Effect of coconut meal on coturnix quail and of coconut meal and coconut oil on performance, carcass measurements and fat composition in swine. J. Anim. Sci., 33, 370-375. 
DEMARNE Y., TOURÉ M., FLANZY J., LECOURTIER M. J., 1974. Evolution des réserves lipidiques du jeune rat en croissance en fonction du temps et de la qualité des lipides ingérés. Ann. Biol. anim. Bioch. Biophys., 14, 793-811.

DEMARNE Y., TOURÉ M., FLANZY J., LECOURTIER M. J., 1975. Influence du degré d'insaturation des lipides alimentaires sur la croissance et la lipogénèse chez le rat. Nutr. Metab., 19, 28-40.

ECKSTEIN M. C., 1929. The influence of diet on the body fat of the white rat. J. biol. Chem., 81, 613-628.

FLANZY J., RÉRAT A., FRANÇOIS A. C., 1968. Etude de l'utilisation digestive des acides gras chez le porc. Ann. Biol. anim. Bioch. Biophys., 8, 537-548.

FLANZY J., FRANÇOIS A. C., RÉRAT A., 1970. Utilisation métabolique des acides gras chez le porc. Ann. Biol. anim. Bioch. Biophys., 10, 603-620.

FOLCH J., LEES M., SLOANE-STANLEY G. H., 1957. A simple method for the isolation and purification of total lipids from tissues. J. biol. Chem., 226, 497-509.

HARLAN W. R., WAKIL S. J., 1963. Synthesis of fatty acids in animal tissues. I. Incorporation of $C_{14}$-acetyl coenzyme $A$ into a variety of long chain fatty acids by subcellular particles. J. biol. Chem., 238, 3216-3223.

HENRY Y., BOURDON D., 1973. Utilisation digestive de l'énergie et des matières azotées de la féverole sous forme entière ou décortiquée, en comparaison avec le tourteau de soja. J. Rech. Porcine en Fronce, INRA-ITP Ed., Paris, 105-114.

HILDITCH T. P., WILLIAMS P. N., 1964. The chemical constitution of natural fats, p. 117, Chapman and Hall Ed., London.

INGR I., 1971. Relation between the topography and fatty acid composition of porcine depot fats Acta vet. Brno, 40, 163-166.

KAUNITZ H., JOHNSON R. E., 1968. Nutritional properties of medium chain triglycerides. J. Am. Oil Chem. Soc., 45, 19-22.

LONGENECKER H. E., 1939. Deposition and utilization of fatty acids of low molecular weight and a fatty acid analysis of coconut oil. J. biol. Chem., 130, 167-177.

MÉTAIS P., BACH A., WARTER J., 1967. Comparaison par l'étude du $14-\mathrm{CO}_{2}$ de l'air expiré de l'utilisation des graisses à acides gras longs, moyens ou courts. C. R. Soc. Biol., 161, 1372-1376.

NUTGEREN D. H., 1965. The enzymic elongation of fatty acids by rat liver microsomes. Biochim. biophys. Acta, 106, 280-290.

PARISOT A., 1949. Constantes et données numériques des corps purs de la chimie des corps gras, 346 p., Dunod Ed., Paris.

POWELL M., 1930. The metabolism of tricaprylin and trilaurin. J. biol. Chem., 89, 547-552.

POWELL M., 1932. The metabolism of tricaprylin. J. biol. Chem., 95, 43-45.

RAULIN J., LAUNAY M., 1964. Conditions permettant la rétention préférentielle d'acide linoléique dans le fissu adipeux du rat incubé in vitro en présence d'adrénaline. C. R. Acad. Sc. Paris 258, 6542-6546.

VISSCHER F. E., 1946. Storage of hendecanoic acid in the white rat. J. biol. Chem., 162, 129-132.

WAKIL S. J., 1961. Mechanism of fatty acid synthesis. J. Lipid Res., 2, 1-24. 